\title{
Insuficiencia cardiaca: la epidemia creciente de una enfermedad multisistémica y de abordaje multidisciplinario
}

\author{
Heart failure: increasing epidemic of a systemic disease with a multidisciplinary approach
}

\author{
Julio Montes Santiago ${ }^{1}$, Alfonso Varela Román² \\ ${ }^{1}$ Servicio de Medicina Interna. Complejo Hospitalario Universitario. Vigo. \\ ${ }^{2}$ Servicio de Cardiología. Complejo Hospitalario Universitario. Santiago.
}

La insuficiencia cardiaca (IC) constituye una epidemia de proporciones colosales, distribución universal y que supone gravosas cargas económicas para los sistemas sanitarios. En efecto, se calcula que en Europa hay de más de 15 millones de personas con dicha enfermedad, 2,4 millones en Japón, 0,3 millones en Australia y constituye el 3-7\% de las hospitalizaciones en África. En EEUU existen 6,6 millones de afectados mayores de 19 años pero se calcula que habrá cerca de 10 millones en 20301.

En 2010 en España se produjeron más de 113000 altas hospitalarias y se calculan cercanas a 150000 las hospitalizaciones para el año 2020. El coste hospitalario calculado actual (2010) es de 473 millones (2,6\% del gasto hospitalario) pero puede superar largamente los 510 millones si se incluyen trasplantes y dispositivos resincronizadores/desfibriladores. El coste real incluyendo seguimiento extrahospitalario y de cuidadores puede llegar a superar los 2200 millones anuales $(\approx 4 \%$ del gasto sanitario global). Por otra parte, 2 de cada 3 pacientes ingresados son $>75$ años. La mortalidad hospitalaria en $>35$ años se sitúa en el 10,4\% y la mortalidad tras la $1^{\text {a }}$ hospitalización puede rondar en torno al $26 \%{ }^{2}$. Estas cifras son muy superiores a las de los cánceres más habituales, por lo que aun puede seguir afirmándose, con razón, que "la insuficiencia cardiaca es más maligna que el cáncer"”.

En cuanto a los pacientes $>35$ años ingresados, cuya media de edad es de 78 años, y en su mayor parte mujeres (56\%), se ha demostrado que el $61 \%$ presentan IC con fracción de eyección preservada (>50\%). Explicando en parte la alta mortalidad y complejidad de su manejo, se registra la frecuente presencia de entidades comórbidas, lo que hacen de la IC una entidad fundamentalmente sistémica: EPOC (35\%), enfermedad cardiovascular y demencia (9\%), insuficiencia renal (13\%), diabetes mellitus (38\%) y fibrilación auricular (48\%)1.

Por fortuna, en los últimos años se ha demostrado que el uso gradual of terapias ajustadas a las Guías se asocia con una apreciable mejoría en la supervivencia ${ }^{4,5}$. Ello resalta la necesidad de implicarse fuertemente en la implementación lo más completa posible de las terapias sugerida en las Guías.

En los últimos tiempos a estas medidas (uso de inhibidores del Enzima conversor de angiotensina/bloqueadores del receptor de angiotensina, implantación de dispositivos cardioversoresdesfibriladores, anticoagulación para la fibrilación auricular y medidas educadoras en IC ${ }^{5}$ y seguramente también el uso de agonistas de aldosterona ${ }^{6}$, se ha unido la ibavradina que ha mostrado aumentar la supervivencia y disminución de hospitalizaciones en $\mathrm{IC}^{7}$.

La presente publicación de actualización sobre la IC, surge como consecuencia de la colaboración de las secciones gallegas de la Sociedad Española de Cardiología y de Medicina interna (SOGACAR y SOGAMI), que son las especialidades mayoritariamente encargadas de su manejo. En España en 2010 el 81\% de pacientes con IC ingresaron a cargo de Servicios de Cardiología o Medicina Interna)(Ministerio de Sanidad, 2010). En la publicación se abordan y actualizan cuestiones de elevado interés como la epidemiología real del paciente con IC en Galicia, su abordaje práctico a partir de las consultas de Cardiología y Unidades de Seguimiento de Medicina Interna, así como la referencia a los últimos datos de los estudios con ibavradina, y que permiten albergar más optimistas perspectivas en esta patología.

Como conclusión, pues, se impone que las características actuales de los pacientes con IC en Galicia requieren un abordaje multidisciplinar con participación de los Servicios de Cardiología y Medicina Interna, abordaje integral, colaborativo entre las diversas especialidades y con un papel primordial desempeñado por Atención Primaria, con abordajes guiados por la evidencia científica que permitan una respuesta adecuada a los crecientes retos de esta epidemia.

\section{Bibliografía}

1. Montes Santiago J. Epidemiología de la insuficiencia cardiaca: aproximación al paciente real en Galicia. Gal Clin 2012; Supl. 3 (en prensa).

2. Stewart S, Macintyre K, Hole De et al. "More malignant tan cancer?. Five year survival following a first admission for heart failure. Eur J Heart Fail 2001; 3: 315-22.

3. Setoguchi S, Stevenson LW. Hospitalizations in patients with heart failure: Who and why. J Am Coll Cardiol 2009; 54: 1703-4.

4. Komajda M, Lapuerta P, Hermans $\mathrm{N}$, et al. Adherence to guidelines is a predictor of outcome in chronic heart failure: the MAHLER survey. Eur Heart J 2005; 26, 1653-59.

5. Fonarow GC, Albert NM, Curtis AB et al. Incremental Reduction in Risk of Death Associated With Use of Guideline-Recommended Therapies in Patients With Heart Failure: A Nested Case-Control Analysis of IMPROVE HF. J Am Heart Assoc 2012; 1:16-26

6. Zannad F, McMurray JJV, Krum H et al.; for the EMPHASIS-HF Study Group. Eplerenone in patients with systolic heart failure and mild symptoms. N Engl J Med, 2011; 364: 11-21.

7. Swedberg K, Komajda M, Böhm M, et al. Effects on outcomes of heart rate reduction by ivabradine in patients with congestive heart failure: is there an influence of betablocker dose? Findings from the SHIFT (Systolic Heart failure treatment with the If inhibitor ivabradine Trial) study. J Am Coll Cardiol 2012; 59:1938-1945. 